\title{
Prognostic significance of the epithelial-to- mesenchymal transition markers e-cadherin, vimentin and twist in bladder cancer
}

Jun Zhao, Dahai Dong, Lingling Sun, Guiming Zhang, Lijiang Sun

Department of Urology, Hospital of the Medical School of Xi-an Jiaotong University (JZ), Xi-an, Shanxi 710061, Department of Urology (DD, GZ, LS) and Department of Pathology (LS), Hospital of the Medical School, Qingdao University, Qingdao, Shandong 266011, P. R. China

\section{ABSTRACT}

Objective: The goal of this study was to utilize long-term patient follow-up to determine whether epithelial-to-mesenchymal transition (EMT)-related markers can predict bladder cancer patient survival and progression of disease.

Materials and Methods: This study included 121 patients with bladder cancer. Sixty-four of these patients presented with non-muscle invasive (NMI, stage T1) bladder cancer and 57 with muscle invasive (MI, stage T2, T3). The patients were diagnosed and treated between May 1998 and July 2012. The EMT markers E-cadherin, Twist, and Vimentin were detected via immunohistochemistry. Univariate and multivariate/Cox analyses were then utilized to determine whether these EMT markers could be useful prognostic markers for predicting bladder cancer patient outcomes.

Results: Analysis of the 121 bladder cancer patients in this study revealed that the frequency of E-cadherin expression was 59.5\% (72/121), Twist was 54.5\% (66/121), and Vimentin was 24.8\% (30/121). Twist and Vimentin were found to have statistically significant correlations with grade, recurrence, and progression but not with stage, whereas E-cadherin was associated with stage but not with the other parameters. In the univariate analysis, grade $(p=0.02)$ was the only significant predictor for progression-free survival (PFS). Stage, grade, and expression of E-cadherin, Vimentin and Twist were included in the multivariate analysis of predicting PFS. In this analysis, grade ( $p$ $=0.01)$ and Vimentin expression $(\mathrm{p}=0.001)$ were found to be significant prognostic factors in predicting PFS.

Conclusions: Grade and Vimentin are potential independent indicators in predicting bladder cancer progression and survival.

\section{ARTICLE INFO}

\section{Key words:}

Urinary Bladder Neoplasms;

Cadherins; Prognosis; Survival

Int Braz J Urol. 2014; 40: 179-89

Submitted for publication:

March 08, 2013

Accepted after revision:

January 07, 2014

\section{INTRODUCTION}

Bladder cancer is the second most common malignancy of the urinary tract with 386,000 cases and 150,000 estimated deaths in the United States in 2011 (1). In Europe, approximately 36,500 men and 13,000 women die of this ma- lignancy every year. Bladder cancer can originate in different parts of the bladder, and patient outcomes can be unpredictably variable. Initially, $70-80 \%$ of patients present with noninvasive (NI) (pTa) or superficially invasive (SI) (pT1) disease whereas 20-30\% are diagnosed with muscle invasive (MI) bladder cancer (pT2-4) (2). Problematic 
inconsistencies and errors exist in the diagnosis and treatment. Over-staging (40\%) and under-staging (nearly 20\%) of clinically localized lesions occur in the diagnosis of bladder cancer (3). Misdiagnosis can lead to improper treatment and an inaccurate prognosis. Therefore, more research is needed to determine how to accurately stage bladder cancer prior to radical cystectomy and to predict the prognosis.

Metastasis is the main cause of death from bladder cancer, and micrometastasis, as the first step of tumor metastasis, appears to be extremely important (4). Epithelial-to-mesenchymal transition (EMT) is a key step during embryonic development and epithelial tumor metastasis (5). EMT causes changes in cell-cell and cell-extracellular matrix interactions resulting in transmigration of cancer cells, thus leading to metastasis $(6,7)$. EMT changes occur at the molecular level before changes in cancer morphology are observed, as has been shown in B-cell lymphoma (8) and melanoma (9); hence utilization of these early molecular changes may be advantageous in predicting the prognosis of bladder cancer (10).

Therefore, to ascertain new prognostic factors, a long-term follow-up study for patients with bladder cancer was conducted whereas the value of EMT biomarkers in predicting prognosis of this malignancy were assessed through the EMT indices E-cadherin, Twist, and Vimentin. E-cadherin is a cell-cell junction protein that is frequently downregulated or lost during EMT, whereas expression of Vimentin and Twist are acquired during this process.

\section{MATERIALS AND METHODS}

\section{Patients' characteristics}

A retrospective review was performed for 121 bladder cancer patients who were diagnosed and treated in the Department of Urology at the Affiliated Hospital of Qingdao University Medical School (P. R. China) from May 1998 to July 2007. The cut-off for follow-up data was July 2012. Some patients with MI underwent radical cystectomy and those with superficially invasive bladder cancer underwent transurethral resection (TUR) followed by prophylactic intravesical che- motherapy. The operative method was selected according to the results of cystoscopy and biopsy in combination with the expressed desires of the patient. Fifty-eight low grade papillary carcinoma patients and 36 high grade papillary carcinoma patients underwent TUR-BT (transurethral resection of bladder tumor), six low grade papillary carcinoma patients and six high grade papillary carcinoma patients underwent partial cystectomy, and three low grade papillary carcinoma patients and 12 high grade papillary carcinoma patients underwent radical cystectomy (RC). Thirty-five of the patients (14 low grade; 21 high grade) who underwent TUR-BT, nine of the patients (four low grade; five high grade) who underwent partial cystectomy, and 13 of the patients (three low grade; 10 high grade) who underwent RC were certified as MI post-operation. Fifty-nine of the patients (44 low grade; 15 high grade) who underwent TUR-BT, three of the patients (two low grade; one high grade) who underwent partial cystectomy, and two of the patients (zero low grade; two high grade) who underwent RC were certified as NMI after operation. All of the patients who were diagnosed with MI bladder cancer after TUR-BT refused subsequent operations. The mean (range) age of the 121 patients was 67 (29-95) years-old. Table- 1 shows the general characteristics of the patients. The tissues were analyzed via immunohistochemistry at the first operation. Tissue slides were reviewed by genito-urinary pathologists. Staging was determined according to the 2002 tumor, lymph nodes, metastasis (TNM) classification of the International Union Against Cancer, and grading was assessed following the 2004 World Health Organization/International Society of Urological Pathology standard.

\section{Immunohistochemistry}

A formalin-soaked, paraffin-encapsulated tissue block was cut into $4 \mu \mathrm{m}$-thick sections, which were placed on slides. Some of the slides were stained with standard haematoxylin and eosin (HEtE). For immunohistochemistry, the paraffin sections were first dewaxed in xylene and rehydrated in decreasing concentrations of alcohol. To restore the antigen, the slides were placed in $0.01 \mathrm{~mol} / \mathrm{L}$ citrate buffer ( $\mathrm{pH}$ 6.0) and heated in 
Table 1 - Clinical and pathological data of the bladder tumors.

\begin{tabular}{|c|c|}
\hline & No. of patients (\%) \\
\hline \multicolumn{2}{|l|}{ Sex } \\
\hline Female & $31(25.6)$ \\
\hline Male & $90(74.4)$ \\
\hline \multicolumn{2}{|l|}{ Multiplicity } \\
\hline Solitary & $95(78.5)$ \\
\hline Multiple & $26(21.5)$ \\
\hline \multicolumn{2}{|l|}{ Tumor size (diameter) } \\
\hline$\leq 3 \mathrm{~cm}$ & $89(73.6)$ \\
\hline$>3 \mathrm{~cm}$ & $32(26.4)$ \\
\hline \multicolumn{2}{|l|}{ Stage (TNM 2002) } \\
\hline pT1 & $64(52.9)$ \\
\hline pT2 & $46(38)$ \\
\hline pT3 & $11(9.1)$ \\
\hline \multicolumn{2}{|l|}{ Grade (WHO 2004) } \\
\hline Papillary carcinoma, low grade & $65(53.7)$ \\
\hline Papillary carcinoma, high grade & $56(46.3)$ \\
\hline \multicolumn{2}{|l|}{ Surgical procedure } \\
\hline TUR & $94(77.7)$ \\
\hline Cystectomy & $\begin{array}{c}27 \text { (12 partial, } 15 \text { total) } \\
(22.3)\end{array}$ \\
\hline \multicolumn{2}{|l|}{ Progression } \\
\hline Total & $41(33.9)$ \\
\hline pT1 & $22(53.7)$ \\
\hline pT2 & $14(34.1)$ \\
\hline pT3 & $5(12.2)$ \\
\hline \multicolumn{2}{|l|}{ Recurrence } \\
\hline Total & $49(40.5)$ \\
\hline pT1 & $18(36.7)$ \\
\hline pT2 & $22(44.9)$ \\
\hline рT3 & $9(18.4)$ \\
\hline \multicolumn{2}{|l|}{ Death } \\
\hline Total & $55(45.5)$ \\
\hline pT1 & $21(38.2)$ \\
\hline pT2 & $24(43.6)$ \\
\hline рT3 & $10(18.2)$ \\
\hline
\end{tabular}

the microwave $\left(100^{\circ} \mathrm{C}\right)$ twice for five min each time. Endogenous peroxidase activity was blocked by placing the slides in 3\% hydrogen peroxide for $15 \mathrm{~min}$. at room temperature. This was followed by three five min washes with phosphate buffered saline (PBS). The tissue sections were placed in a $4^{\circ}$ $\mathrm{C}$ humidified chamber with primary antibody. The rabbit polyclonal E-cadherin antibody, the goat polyclonal Twist antibody and the mouse monoclonal Vimentin antibody (Santa Cruz Biotechnology, Santa Cruz, CA, USA) were diluted 1:200, $1: 100$ and $1: 100$, respectively. After washing with PBS, the sections were incubated with secondary antibody (Zhongshan Golden Bridge Biotechnology, Beijing, China) for $30 \mathrm{~min}$. and washed twice for five min with PBS. The sections were stained with diaminobenzidine tetrahydrochloride, then counterstained with haematoxylin, dehydrated, and covered with a cover slip. For the negative control, the primary antibody was replaced with PBS. The positive control was supplied with the kit (Santa Cruz Biotechnology).

\section{Digital image analysis}

All samples were reviewed by an independent investigator who was blinded to the clinical outcomes of the patients. The stained sections were reviewed using an Olympus microscope (Olympus Corporation, Tokyo, Japan) and digital images were captured and processed in TIFF format. Image Pro Plus 6.0 (Media Cybernetics Inc., Bethesda, MD, USA) was used to calculate the intensity of the detected molecules. Six microscopic fields within the tumor tissue sections (original magnification 40X) were randomly selected and the integral optical density (IOD) was calculated for each image. All IOD values were divided into four quartiles as follows: $0-25 \%$ negative expression, 25-50\% weak expression, 50-75\% moderate expression, and $75-100 \%$ strong expression. The sample was considered to be positive when the IOD $\geq 50 \%$ (11).

\section{Statistical analysis}

The survival time of patients who underwent TUR, partial cystectomy or RC for different-stage bladder cancer was analyzed. The time 
points for TUR or partial cystectomy were from the last surgery to either the date of recurrence, date of progression or the date of final follow-up, whereas the time points for RC were from the operation date to either the date of death or the date of the last follow-up.

Overall survival (OS) was defined as from the day of surgery to either the day of death from any cause or censored at the time of the last follow-up, whereas progression-free survival (PFS) was defined as from the day of surgery to either the day of progression of the cancer or to the day of follow-up termination. Increases in T stage or in grade (from low to high) were considered indicators of mean progression. The Chi-squared test was used to determine the correlation between Vimentin, Twist and E-cadherin expression and sex, pathology, stage and grade. One-factor analysis was performed using the log-rank test. The Kaplan-Meier method was utilized and Kaplan-Meier curves were constructed. A multivariable analysis was performed using the Cox proportional hazard regression model. $\mathrm{P}<0.05$ was considered to be statistically significant. All of the tests were two-sided. Statistical Product and Service Solutions version 17.0 (SPSS Inc., Chicago, IL, USA) was used for data analysis.

\section{RESULTS}

One-hundred-and-twenty-one patients were included in this study with a median follow-up time ranging from 60-180 months (median of 72 months). Fifty-five of the patients (45.5\%) died during the follow-up period, 49 (40.5\%) recurred, and 41 (33.9\%) progressed (Table-1). Seventy-two (59.5\%) of the 121 tissue specimens examined were positive for E-cadherin expression via immunohistochemical analysis, 66 (54.5\%) were positive for Twist, and $30(24.8 \%)$ were positive for Vimentin (Table-2 and Figure-1). The IOD of E-cadherin revealed higher expression in low grade papillary carcinoma $(159105 \pm 42036)$ than in high grade papillary carcinoma (15369 \pm 4762), whereas Vimentin (17262 \pm 5942 low grade, 71056 \pm 27324 high grade) and Twist (24996 \pm 7764 low grade, $86991 \pm 19683$ high grade) demonstrated the opposite expression pattern.
Three patients had previous TUR in a different hospital (3, 4 and 8 months, respectively) before admission to our hospital to undergo RC for recurrent bladder cancer. Their molecular profiles were E-cadherin negative, Twist positive, and Vimentin positive. These patients died at six, nine, and 10 months, respectively, after surgery in our hospital. These results are consistent with high grade papillary carcinoma having undergone EMT, thus losing expression of E-cadherin and gaining expression of the EMT markers Vimentin and Twist.

The Chi-squared test was used to evaluate the correlation of Vimentin, Twist, and E-cadherin expression with sex, pathology, stage, grade, recurrence and progression. The expression of both Twist and Vimentin were found to have statistically significant correlations with grade, recurrence and progression. In contrast, E-cadherin expression was found to have a statistically significant correlation with stage (Table-2, Figure-1). Among the patients diagnosed with pT2 bladder cancer, 22 patients recurred, 14 patients progressed, and 24 patients died. Sixty-eight-point-two percent (15/22) of the patients who recurred had tumors that stained positively for E-cadherin and 56.5\% (13/23) were E-cadherin negative, 42.8\% (6/14) of the patients who progressed had E-cadherin positive tumors compared to $68.8 \%(22 / 32)$ E-cadherin negative, and 58.3\% (14/24) of the patients who died had E-cadherin positive tumors whereas 59.1\% (13/22) were E-cadherin negative. Sixty-eight-point-two percent of the patients who recurred had tumors positive for Twist and 26.1\% (6/23) were Twist negative, 92.9\% (13/14) of the patients who experienced disease progression had Twist positive tumors whereas 46.9\% (15/32) were Twist negative, and 66.7\% (16/24) of patients who died had Twist positive tumors compared to 45.5\% (10/22) that were negative. Finally, 36.4\% (8/22) of patients who recurred had Vimentin positive tumors with $17.4 \%$ (4/23) being negative, 64.3\% (9/14) of patients who progressed had tumors positive for Vimentin whereas 21.9\% (7/32) were Vimentin negative, and 41.7\% (10/24) patients who died had Vimentin positive tumors compared to 27.3\% (6/22) that were Vimentin negative. E-cadherin, Twist and Vimentin expression exhibited statistically significant differences in the recurrence 


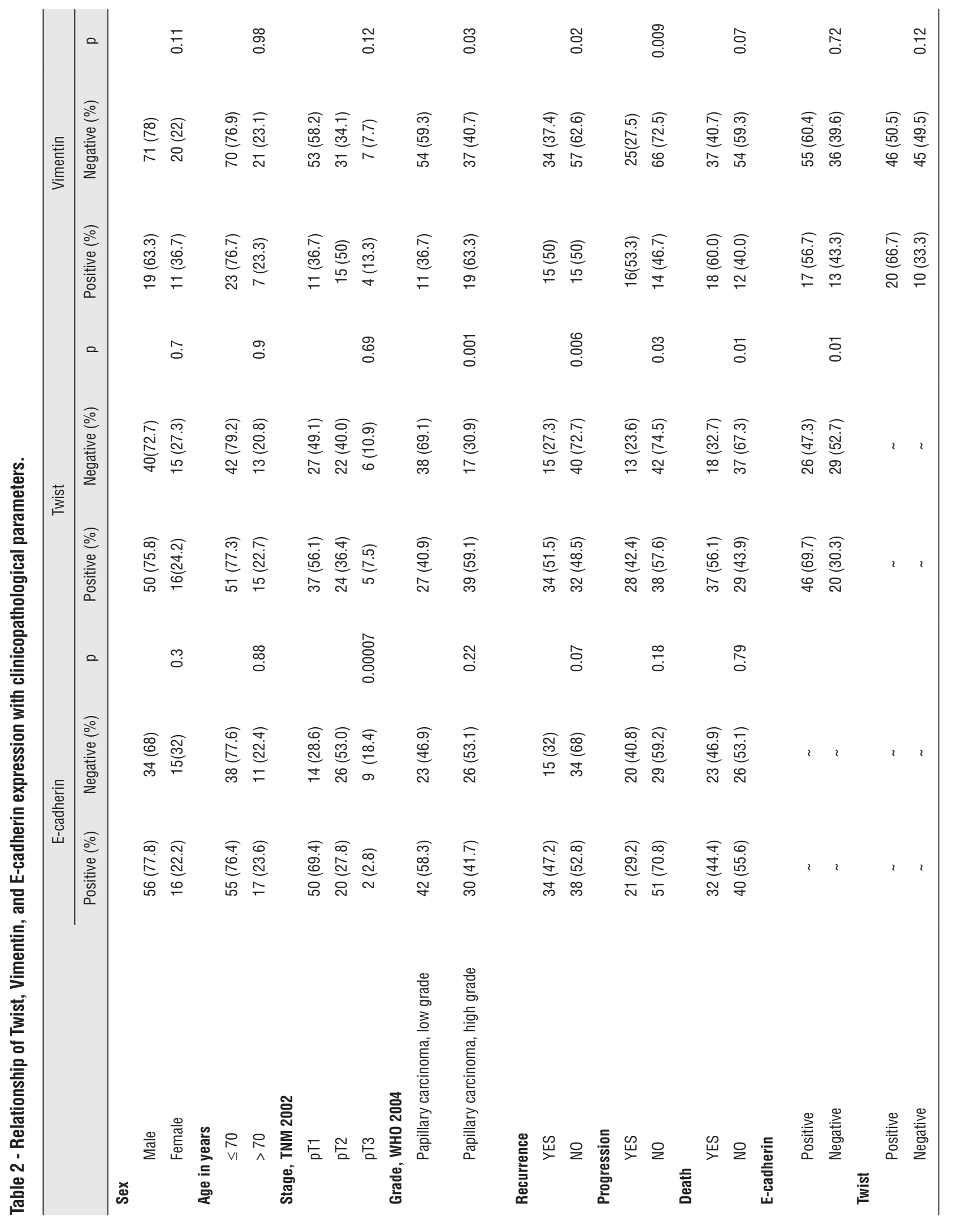


Figure 1 - E-cadherin, Twist and Vimentin expression in bladder cancer. A. Images of immunohistochemically detected expression. B. Bar graph of the values of the integrated optical density of E-cadherin, Twist and Vimentin in low and high grade papillary carcinoma. Results are expressed as the mean \pm SD.

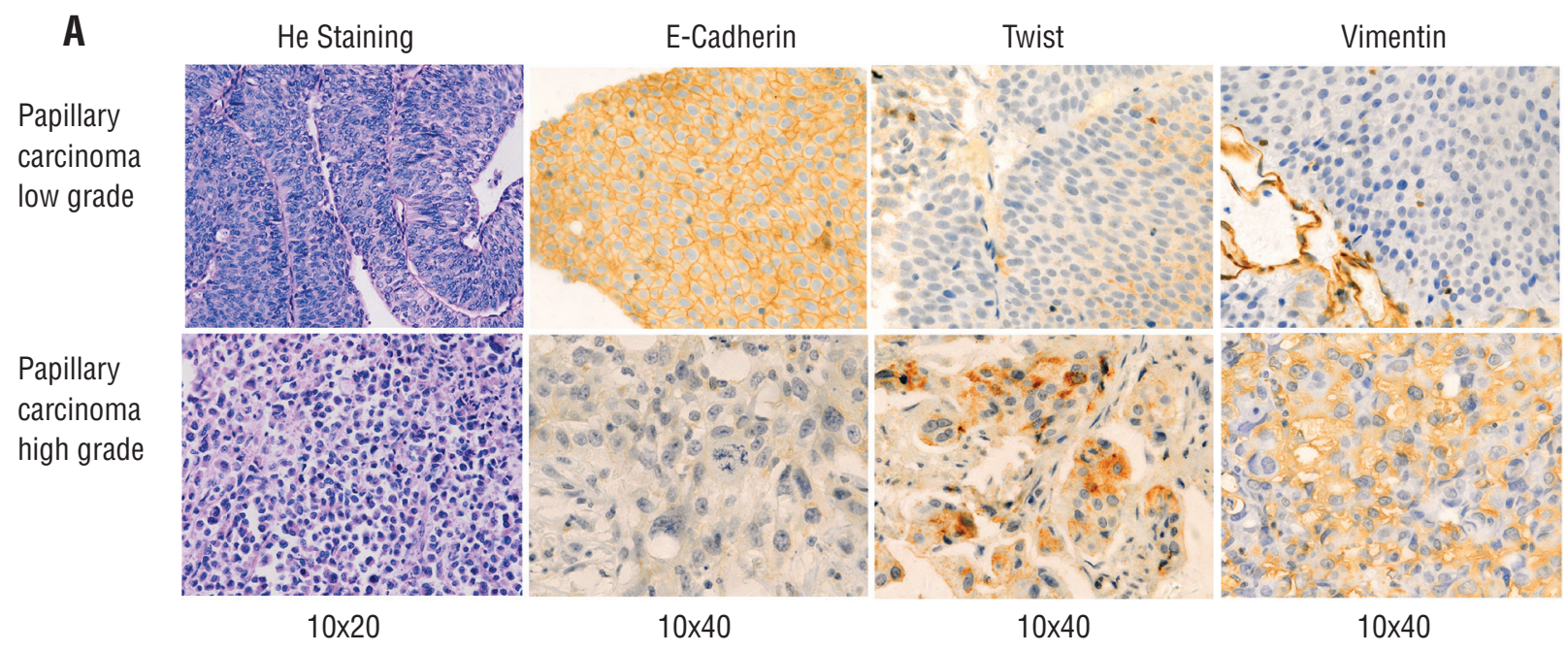

B

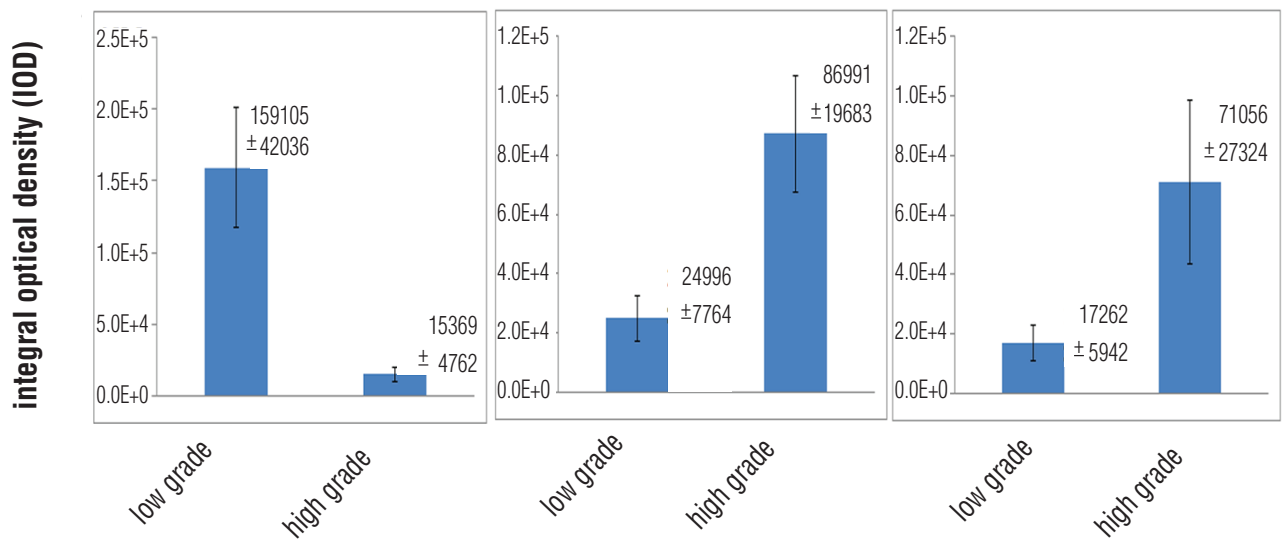

versus non-recurrence, progression versus non-progression, and death versus no death groups, with the exception of E-cadherin expression in the progression versus non-progression group (Table-3).

In the univariate analysis, the time point for PFS/OS was set for 60 months and grade was found to be the only significant predictor for PFS. The others indices were not found to be significant predictors of PFS and OS (Table-4, Figure-1). To further investigate the relationship between survival and molecular profile and abate the inter- -effect among the indices, Cox regression analysis was performed using the following parameters: stage, grade and expression of Twist, E-cadherin and Vimentin. Grade $(p=0.01)$ and Vimentin ( $p$ $<0.001$ ) expression were found to be significantly correlated with PFS in this analysis.

\section{DISCUSSION}

Bladder cancer is a common malignancy with an estimated 70,530 new cases in the United 


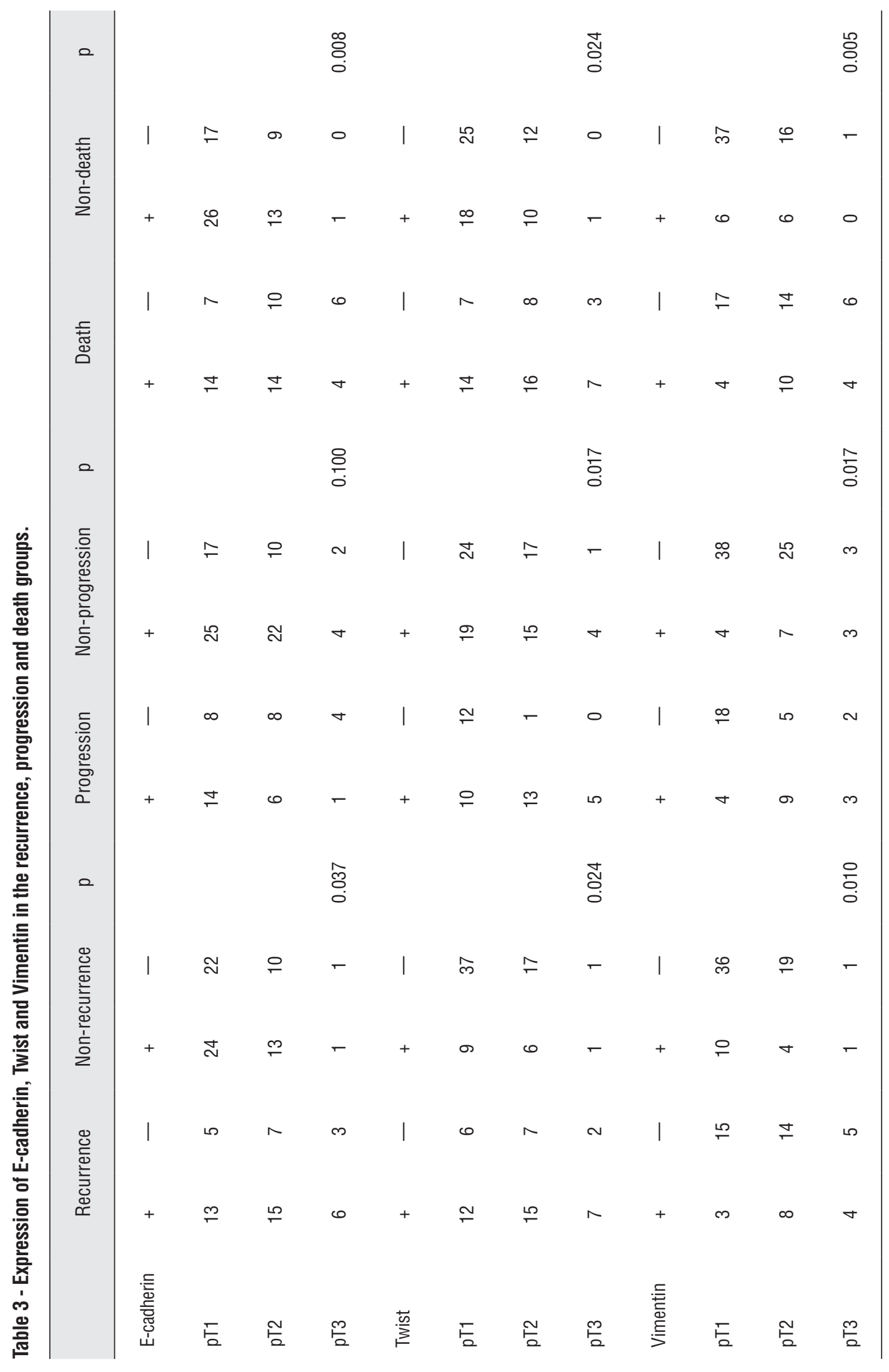




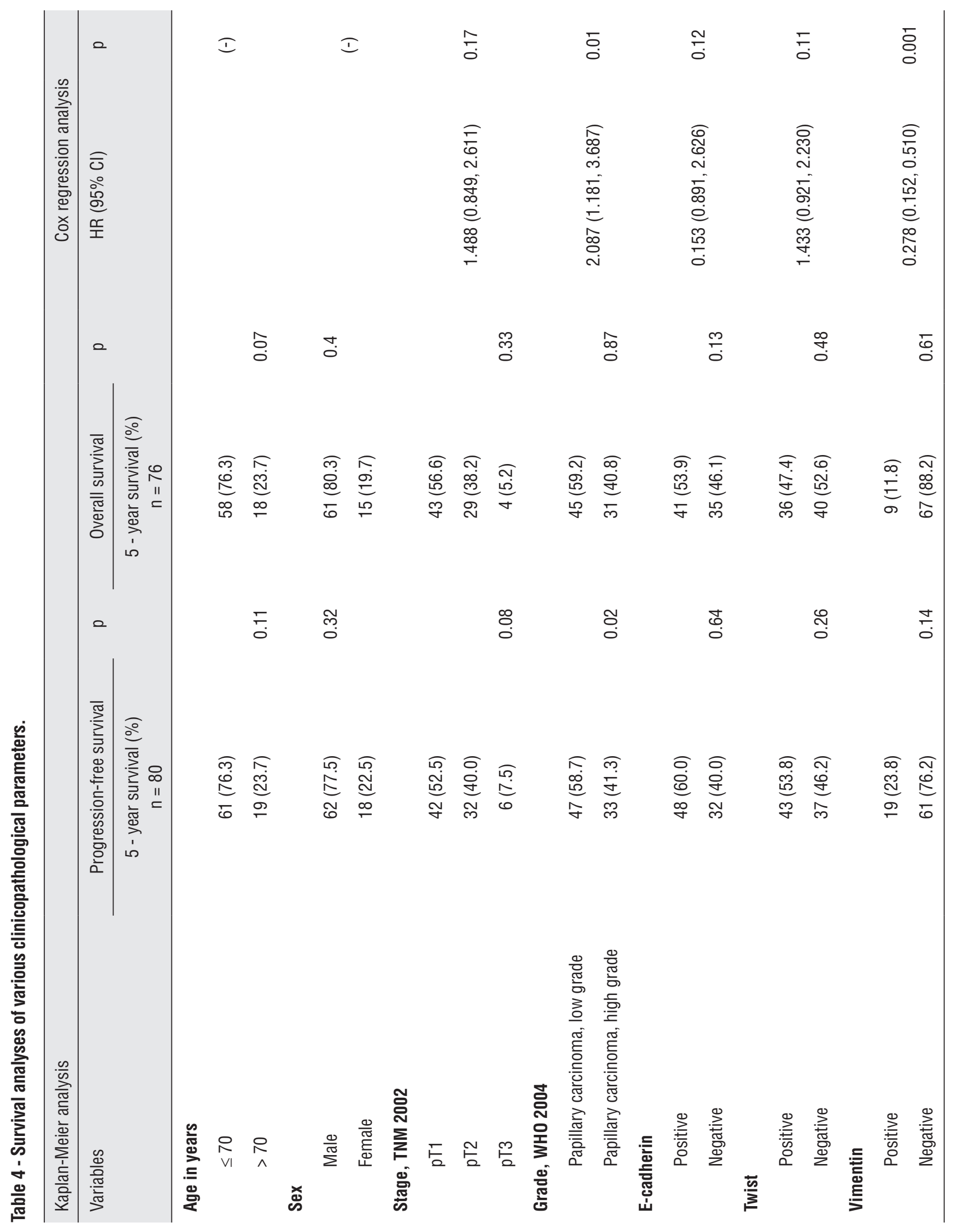


States in 2010 (12). Most of the tumors initially present as NI or SI (i.e. pTa or pT1), so TUR is performed. Over $70 \%$ of these cases recur and require re-operation, of which, about 10-15\% will advance to MI (13). Therefore, it is necessary to be able to accurately stage and predict the prognosis of this malignancy.

Molecular diagnostics are routine clinical practice in patients with lung, colon, and breast cancer. Bladder cancer, a heterogeneous disease with diverse genetic and environmental risk factors, currently lacks useful molecular biomarkers. Therefore, identifying biomarkers that can accurately predict recurrence, progression and outcome are needed. It is important that we find and validate prognostic molecular biomarkers that can help clinicians to identify patients in need of early, aggressive management $(14,15)$.

Errors in clinical staging and pathological grading of this malignancy frequently occur, thus procedures for staging and grading need to be improved (16). One problem that plays a role in bladder cancer staging is burning of the tissue during cauterization. Tissue burnt during electro-resection makes it very difficult to determine whether the stroma or superficial muscular layer of the bladder has been invaded, thus complicating staging of the cancer. To avoid this, energy during electrotomy should be kept to a minimum (17). To cure a patient with primary NMI bladder cancer, a high quality TUR that removes all (pre)malignant lesions must be performed (18). Over-staging typically occurs if muscle tissue is not included in the first-time excisional specimens of a T1 lesion (19). Computed tomography and magnetic resonance imaging cannot discriminate the various layers (lamina propria, superficial and deep muscle) of the bladder, therefore clinical classification of stages T1, T2a and T2b is nearly impossible (20). Due to the limitations of classic clinicopathological indices and imaging manifestations, other prognostic factors (carcinoma in situ, hydronephrosis) and molecular markers that are under investigation are promising as repeatable and predictive factors in the diagnosis of bladder cancer $(21,22)$. Our data support the use of such molecular markers in more accurately diagnosing bladder cancer. Among bladder cancers, pT2 is the most commonly mis- diagnosed stage. In the current study, pT2 was the diagnosis in $44.9 \%(22 / 49)$ of patients in the recurrence group, in 34\% (14/41) of patients in the progression group, and 43.6\% (24/55) of patients in the death group, thus accounting for a significant proportion of the diagnoses. Moreover, in the progression group, pT1 + pT2 accounted for $87.8 \%$ of the diagnoses. In the recurrence, progression and death groups for bladder cancer patients diagnosed with pT2 bladder cancer, Twist was positive in $68.2 \%, 92.9 \%$, and $66.7 \%$ of patient tumors, respectively, and Vimentin was positive in $36.4 \%$, $64.3 \%$, and $41.7 \%$ of patient tumors, respectively. The Twist and Vimentin positive expression in these patients was significantly higher than that in the patients who were negative for Twist and Vimentin in these three groups. E-cadherin expression in the recurrence, progression and death groups demonstrated an expression pattern that was opposite to that of Twist and Vimentin. Our data demonstrate that molecular changes in bladder cancer tissue are not significantly affected by outside factors (such as the method of operation or the experience of the operator) and more objectively reflect the characteristics of the cancer.

In the current study, stage, grade and expression of three EMT-related molecular markers were compared during the 60-180 month follow-up period. Grade was found to be more effective than stage in predicting progression and recurrence, and it correlates more efficiently with tumor markers than stage. This may be because diagnosis of stage is more prone to error due to cauterization of tissue than the diagnosis of grade.

EMT is a critical step during cancer progression in which downregulation or loss of E-cadherin expression (epithelial marker) occurs, making E-cadherin a potential biomarker. Downregulation or loss of E-cadherin is often accompanied by upregulation of N-cadherin, Twist, and Vimentin (mesenchymal markers) during EMT, thus making these factors attractive potential markers also (23). Various EMT related markers are used as prognostic markers in other types of cancers $(7,10,24,25)$. In this study, the relationships between three EMT-related molecular markers and five year PFS and five year OS were investigated. Twist and Vimentin were significantly correlated 
with grade, progression, and recurrence, suggesting that these two factors would be useful biomarkers in the diagnosis and treatment of bladder cancer patients. Patients with NMI bladder cancer and expression of Twist or Vimentin would be treated with a more aggressive therapy regimen to prevent progression and recurrence.

Down-regulation or loss of E-cadherin is one of the initial molecular events in the process of EMT. This is followed by major changes in the cytoskeleton that enables cells to acquire a mesenchymal phenotype and subsequent increased motility and invasiveness. Cells undergoing EMT frequently acquire the expression of Vimentin and Twist. (26-28). The IOD of E-cadherin is higher in low grade papillary carcinoma than high grade papillary carcinoma, whereas Vimentin and Twist expression are lower in the low grade cancer and higher in the high grade papillary carcinoma. Khorrami et al. (29) examined E-cadherin expression in 180 patients with unifocal, superficial, low-grade, papillary transitional cell carcinoma of the bladder and followed these patients for 36 months after surgery. E-cadherin immunoexpression was negative in 101 (56\%) and positive in 79 (44\%) patients. Additionally, downregulation of E-cadherin was associated with disease recurrence in NMI bladder cancer. Our results show a similar trend whereby the IOD of E-cadherin in low grade papillary carcinoma was significantly higher than E-cadherin levels in high grade disease. In patients who experienced disease progression or died, E-cadherin expression was largely negative, while Twist and Vimentin expression was mostly positive. In the patients who recurred, no significant difference existed in E-cadherin expression; however, Twist and Vimentin remained primarily positive. These data support the idea that E-cadherin is down-regulated during EMT whereas Twist and Vimentin are up-regulated.

The limitations of the present study are that it is retrospective and immunohistochemical in nature. Clinical application of immunohistochemistry is limited by the following factors: discrepancies between different antibodies, diversity in interpretation and judgment, and inconsistency in specimen preparation and technical procedures. Another potential limitation of our study is that the different predictive value of staining between the nucleus and cytoplasm of E-cadherin, Twist, and Vimentin was not compared. Nonetheless, the results from this study indicate that these markers of EMT should be further investigated as markers to assist in the diagnosis, staging and treatment decisions for bladder cancer patients.

\section{CONCLUSIONS}

Bladder cancer is a multi-origin disease with variable outcomes. Despite the substantial advances made in biomedical research, the error of clinical grading and staging of bladder cancer still exists. Therefore, additional predictors such as molecular markers would be beneficial in improving the accuracy of staging and grading this malignancy. Our study shows that Vimentin has the potential to become an independent predictor for cancer progression and survival. In addition, we find that bladder tumor grade is better than stage at predicting progression and recurrence.

\section{CONFLICT OF INTEREST}

None declared.

\section{REFERENCES}

1. Jemal A, Bray F, Center MM, Ferlay J, Ward E, Forman D: Global cancer statistics. CA Cancer J Clin. 2011; 61: 69-90. Erratum in: CA Cancer J Clin. 2011; 61: 134

2. van Rhijn BW, Burger M, Lotan Y, Solsona E, Stief CG, Sylvester RJ, et al.: Recurrence and progression of disease in non-muscle-invasive bladder cancer: from epidemiology to treatment strategy. Eur Urol. 2009; 56: 430-42.

3. Shariat SF, Palapattu GS, Karakiewicz PI, Rogers CG, Vazina A, Bastian PJ, et al.: Discrepancy between clinical and pathologic stage: impact on prognosis after radical cystectomy. Eur Urol. 2007; 51: 137-49; discussion 149-51.

4. Wallerand H, Robert G, Pasticier G, Ravaud A, Ballanger $P$, Reiter RE, et al.: The epithelial-mesenchymal transitioninducing factor TWIST is an attractive target in advanced and/or metastatic bladder and prostate cancers. Urol Oncol. 2010; 28: 473-9.

5. Thiery JP, Acloque H, Huang RY, Nieto MA: Epithelialmesenchymal transitions in development and disease. Cell. 2009; 139: 871-90. 
6. Ghoul A, Serova M, Astorgues-Xerri L, Bieche I, Bousquet G, Varna M, et al.: Epithelial-to-mesenchymal transition and resistance to ingenol 3-angelate, a novel protein kinase $\mathrm{C}$ modulator, in colon cancer cells. Cancer Res. 2009; 69: 4260-9.

7. Nijkamp MM, Span PN, Hoogsteen IJ, van der Kogel AJ, Kaanders $\mathrm{JH}$, Bussink J: Expression of E-cadherin and vimentin correlates with metastasis formation in head and neck squamous cell carcinoma patients. Radiother Oncol. 2011; 99: 344-8.

8. Lemma S, Karihtala P, Haapasaari KM, Jantunen E, Soini Y, Bloigu $R$, et al.: Biological roles and prognostic values of the epithelial-mesenchymal transition-mediating transcription factors Twist, ZEB1 and Slug in diffuse large B-cell lymphoma. Histopathology. 2013; 62: 326-33.

9. Weiss MB, Abel EV, Mayberry MM, Basile KJ, Berger AC, Aplin AE: TWIST1 is an ERK1/2 effector that promotes invasion and regulates MMP-1 expression in human melanoma cells. Cancer Res. 2012; 72: 6382-92.

10. Hur K, Toiyama Y, Takahashi M, Balaguer F, Nagasaka T, Koike J, et al.: MicroRNA-200c modulates epithelial-tomesenchymal transition (EMT) in human colorectal cancer metastasis. Gut. 2013; 62: 1315-26.

11. Fan HX, Li HX, Chen D, Gao ZX, Zheng JH: Changes in the expression of MMP2, MMP9, and CollV in stromal cells in oral squamous tongue cell carcinoma: relationships and prognostic implications. J Exp Clin Cancer Res. 2012; 31: 90.

12. Jemal A, Siegel R, Xu J, Ward E: Cancer statistics, 2010. CA Cancer J Clin. 2010; 60: 277-300. Erratum in: CA Cancer J Clin. 2011; 61: 133-4.

13. Salinas-Sánchez AS, Lorenzo-Romero JG, Giménez-Bachs JM, Sánchez-Sánchez F, Donate-Moreno MJ, Rubio-DelCampo A, et al.: Implications of p53 gene mutations on patient survival in transitional cell carcinoma of the bladder: a long-term study. Urol Oncol. 2008; 26: 620-6.

14. Netto GJ: Molecular biomarkers in urothelial carcinoma of the bladder: are we there yet? Nat Rev Urol. 2011; 9: 41-51.

15. Chang DW, Gu J, Wu X: Germline prognostic markers for urinary bladder cancer: obstacles and opportunities. Urol Oncol. 2012; 30: 524-32.

16. Bostrom PJ, van Rhijn BWG, Fleshner N, Finelli A, Jewett $M$, Thoms J, et al.: Staging and Staging Errors in Bladder Cancer. Eur Urol Suppl. 2010; 9: 2-9.

17. Babjuk M: Transurethral resection of non-muscle-invasive bladder cancer. Eur Urol Suppl. 2009; 8: 542-8.

18. Herr HW, Donat SM: A re-staging transurethral resection predicts early progression of superficial bladder cancer. BJU Int. 2006; 97: 1194-8.

19. van Rhijn BW: Combining molecular and pathologic data to prognosticate non-muscle-invasive bladder cancer. Urol Oncol. 2012; 30: 518-23.
20. Liu JJ, Droller MJ, Liao JC: New optical imaging technologies for bladder cancer: considerations and perspectives. J Urol. 2012; 188: 361-8.

21. $\mathrm{Ng}$ CS: Radiologic diagnosis and staging of renal and bladder cancer. Semin Roentgenol. 2006; 41: 121-38.

22. van Rhijn BW, Zuiverloon TC, Vis AN, Radvanyi $F$, van Leenders GJ, Ooms BC, et al.: Molecular grade (FGFR3/MIB1) and EORTC risk scores are predictive in primary nonmuscle-invasive bladder cancer. Eur Urol. 2010; 58: 433-41.

23. Gasparotto D, Polesel J, Marzotto A, Colladel R, Piccinin S, Modena P, et al.: Overexpression of TWIST2 correlates with poor prognosis in head and neck squamous cell carcinomas. Oncotarget. 2011; 2: 1165-75.

24. Kallergi G, Papadaki MA, Politaki E, Mavroudis D, Georgoulias V, Agelaki S: Epithelial to mesenchymal transition markers expressed in circulating tumour cells of early and metastatic breast cancer patients. Breast Cancer Res. 2011; 13: R59.

25. Nozato M, Kaneko S, Nakagawara A, Komuro H: Epithelialmesenchymal transition-related gene expression as a new prognostic marker for neuroblastoma. Int J Oncol. 2013; 42: $134-40$.

26. Markiewicz A, Ahrends $T$, Wełnicka-Jaśkiewicz $M$, Seroczyńska B, Skokowski J, Jaśkiewicz J, et al.: Expression of epithelial to mesenchymal transition-related markers in lymph node metastases as a surrogate for primary tumor metastatic potential in breast cancer. J Transl Med. 2012; 10: 226.

27. Moreno-Bueno G, Peinado H, Molina P, Olmeda D, Cubillo E, Santos $V$, et al.: The morphological and molecular features of the epithelial-to-mesenchymal transition. Nat Protoc. 2009; 4: 1591-613.

28. Xie D, Gore C, Liu J, Pong RC, Mason R, Hao G, et al.: Role of DAB2IP in modulating epithelial-to-mesenchymal transition and prostate cancer metastasis. Proc Natl Acad Sci U S A. 2010; 107: 2485-90.

29. Khorrami MH, Hadi M, Gharaati MR, Izadpanahi MH, Javid A, Zargham M: E-cadherin expression as a prognostic factor in transitional cell carcinoma of the bladder after transurethral resection. Urol J. 2012; 9: 581-5.

Correspondence address: Lijiang Sun, MD

Department of Urology, The Affiliated Hospital of the Medical School, Qingdao University, Qingdao, Shandong Jiangsu Road 16\#, 266011, P. R. China Telephone: + 860532 8291-3056 E-mail: sunlijiangdoc@163.com 